Miszalska, J. (2021). Padova nei periodici polacchi tra la fine del XIX sec. e il 1939. Italica Wratislaviensia, 12(1), 161-175.

DOI: http://dx.doi.org/10.15804/IW.2021.12.1.09

Jadwiga Miszalska

Uniwersytet Jagielloński w Krakowie, Polonia

jadwiga.miszalska@uj.edu.pl

ORCID: 0000-0001-7122-9396

\title{
PADOVA NEI PERIODICI POLACCHI TRA LA FINE DEL XIX SEC. E IL 1939
}

\section{PADUA IN THE POLISH PRESS BETWEEN THE LAST DECADES OF THE 19 ${ }^{\mathrm{TH}}$ CENTURY AND 1939}

\begin{abstract}
Italy, seen as the cradle of European culture and the destination of the wanderings of Polish intellectuals and artists, often appears in the Polish press of the late- $19^{\text {th }}$ and early- $20^{\text {th }}$ centuries. Among the cities described, Padua is present, although it appears less frequently than Venice or Rome. Articles dedicated to this city, however, have a particular character, because not only is "Padova la dotta" famous for the cult of Saint Anthony, but it is most often presented in the contexts of centuriesold Italian-Polish relations linked mostly to the University and of the Paduan polonica. There are in fact two moments that find a particular resonance in the Polish press: the first, in 1922, for the seventh centenary of the University, and the second, in 1931, for the celebrations of the seventh centenary of Saint Anthony's death. This article offers a review of the texts that have appeared in various periodicals and includes a brief presentation of the authors - scholars, artists, or journalists active in the promotion of Italian culture. From the texts published both in newspapers and in cultural magazines or even scientific periodicals, the image of the city emerges as strongly marked by the presence of Poles, who were students, university professors, or pilgrims.
\end{abstract}

Keywords: Padua, university, Polish press, Italian-Polish relations, Basilica of St. Anthony 
$\mathrm{V}$ erso la fine del XIX secolo in Europa cresce vistosamente la funzione sociale della stampa periodica. Oltre a numerose riviste di cultura e periodici specialistici bisogna notare l'importanza dei quotidiani, accessibili ad ampie cerchie di pubblico grazie al prezzo non elevato, non solo come fonte di informazioni sulle attualità socio-politiche, ma anche come strumento di educazione culturale. Infatti, gran parte dei quotidiani, oltre a notizie sui fatti correnti della realtà nazionale ed estera nella rubrica feuilleton, propone testi narrativi a puntate, articoli su tematiche culturali o brevi "reportages" dall'estero, che hanno spesso la forma di "lettere da...". D'altra parte nei settimanali o mensili vengono pubblicati testi di dimensioni più ampie e di più grande spessore ${ }^{1}$.

Per la mia ricerca di testi dedicati a Padova e pubblicati sulla stampa polacca dagli ultimi decenni del XIX secolo fino allo scoppio della II Guerra mondiale mi sono servita della bibliografia dei periodici polacchi del XIX e XX secolo accessibile ora online sul sito della Biblioteca Nazionale di Varsavia e dell'Istituto di Studi Letterari dell'Accademia Polacca delle Scienze, la cosiddetta "Bibliografia di Bar"2. Adam Bar fu bibliografo, editore e studioso di letteratura, autore di numerose pubblicazioni di carattere bibliografico, per 30 anni legato alla Biblioteca Jagellonica di Cracovia, il quale ai tempi dell'occupazione nazista iniziò un lavoro certosino sulla bibliografia dei contenuti dei periodici polacchi. Il lavoro, portato avanti insieme agli studiosi della Biblioteca fino al 1953, fu continuato in seguito da quelli dell'Accademia delle Scienze.

Benché la bibliografia, basata su fonti accessibili all'epoca soprattutto nelle biblioteche di Cracovia, non prenda in considerazione tutti i periodici polacchi e presenti alcune lacune nelle singole annate, costituisce comunque un valido strumento di ricerca, comprendendo la stragrande maggioranza della stampa polacca. I dati ricavati dalla suddetta bibliografia sono stati da me completati tramite ulteriori ricerche bibliografiche, il che permette di giudicarli come uno specchio verosimile

1 Per un'analisi approfondita delle funzioni dei periodici polacchi a cavallo tra l' 800 e il '900 e della rubrica feuilleton che ospitava non solo romanzi a puntate vedi p. es. Pietrzak, 2017.

${ }^{2}$ http://bar.ibl.waw.pl/cgi-bin/makwww.exe?BM=1. 
della realtà fattuale in cui il tema dell'Italia si presenta come assai frequente. I quotidiani segnalano puntualmente eventi politici italiani, quali le perturbazioni dei governi Crispi o i moti sociali della fine dell' 800 . L'Italia è inoltre fortemente presente nei settimanali di cultura, come testimoniano numerose notizie sulle novità editoriali non solo di carattere letterario, ma anche di spessore scientifico, resoconti di spettacoli operistici o di mostre d'arte ${ }^{3}$. Un gruppo cospicuo di articoli è costituito dalle corrispondenze dall'Italia, con attenzione rivolta alle singole città, alla loro storia e ai loro monumenti.

Il periodo da me analizzato si racchiude tra due date: la prima menzione di Padova da me ritrovata proviene dal 1878, mentre l'anno 1939, inizio dell'occupazione nazista, segna la fine della stampa libera polacca. Bisogna comunque notare che fino al 1918 la Polonia non esiste come stato politicamente indipendente e le sue diverse regioni fanno parte dei tre grandi imperi: Russia, Prussia ed Austria; malgrado ciò non si sono notate differenze per le scelte dei temi nel materiale analizzato. Gli articoli pubblicati nella Polonia libera dopo la fine della Grande Guerra sono comunque più numerosi che non in quel primo periodo ovvero nell'ultimo quarto dell' 800 e negli anni prebellici, il che può essere risultato della generale crescita del mercato dei periodici. Si nota invece praticamente la mancanza (tranne uno) dei testi nei quattro anni della guerra.

Tra le corrispondenze dall'Italia nel periodo preso in considerazione che riguardano singole città, in posizione privilegiata si trova ovviamente Roma. La bibliografia di Bar segnala circa 350 articoli e note dedicati a questa città. Il secondo posto spetta a Venezia, con circa 90 testi, e il terzo a Napoli, menzionata una quarantina di volte. Poi seguono Padova, Bologna e Assisi, con un numero paragonabile di articoli. Oltre ai 19 articoli dedicati a Padova (uno dei quali non si è trovato forse per dati bibliografici erronei), sono riuscita a rintracciarne altrove altri 12 , arrivando al numero totale di 31 .

3 Un esempio di tale interesse può essere indicato nell'attività del settimanale Prawda (cf. Miszalska, 2020). 
I primi testi pubblicati nell' 800 prendono come tema Padova in quanto centro del culto di sant'Antonio. Il primo che menziona la Basilica del Santo, pubblicato sulla rivista dedicata "al popolo e alla gioventù" Chata, è una poesia del sacerdote Władysław Jachimowski, la quale costituisce una parte del ciclo dedicato al pellegrinaggio italiano del 1877 la cui meta erano anche altre località, tra cui ovviamente Roma. La Basilica del Santo è anche tema di una breve nota anonima pubblicata nel 1895 sul quotidiano Kurier Codzienny. Più esauriente risulta l'articolo del 1892, San Antonio di Padova (z notatek turysty), stampato sul quotidiano Kurier Warszawski e firmato da Czesław Jankowski.

La lettura dei testi dedicati a Padova non lascia dubbi. La città veneta, nominata "Padova la dotta", appare per lo più nel contesto delle relazioni italo-polacche. L'asse intorno al quale, a partire dagli anni ' 80 , si sviluppano gli argomenti toccati, riguarda l'Università e le tracce delle presenze polacche nell'Ateneo e nella città. Infatti, il settimanale di cultura Kłosy pubblica il 26 febbraio del 1885 l'articolo di Adam Pług, Polacy w Akademii Padewskiej. L'autore, come fonte delle sue informazioni sull'Università e sui famosi studenti iscritti alla natio polacca, cita un opuscolo di Aleksander Przeździecki, O Polakach w Bononii i Padwie, edito nel 1853. Il testo è accompagnato da due illustrazioni, una delle quali rappresenta lo schizzo della facciata dell'Ateneo del 1595 e l'altra i portici del cortile nello stato attuale. Kłosy, che negli anni 1886-1887 presentava ai suoi lettori disegni di diversi luoghi italiani - per lo più legati alla storia polacca - eseguiti dal famoso pittore Aleksander Gierymski, il $1^{\circ}$ aprile 1886 stampa il suo disegno del portone principale dell'Università con una breve nota che ricorda l'articolo di Pług pubblicato l'anno precedente. Inoltre nel numero del 7 aprile 1887 vi ritroviamo ancora una puntata della corrispondenza dall'Italia di Cezary Polewka, Listy włoskie, in cui si menziona Jan Kochanowski, accompagnata dal disegno dell'antico altare polacco della basilica del Santo e in quello del 20 ottobre gli schizzi di due lapidi dedicate a Copernico all’Università di Bologna e, appunto, all'Università di Padova, sempre di mano di Gierymski ${ }^{4}$.

${ }^{4}$ Informazioni sulle pubblicazioni in Kłosy da J. Zarzycki, Artysta oraz miejsca pamięci. Padwa, Wenecja (i nie tylko) w prasowych ilustracjach Aleksandra Gierym- 
Negli stessi anni, sul mensile di Cracovia Przeglad Polski apparvero due importanti testi di Stanisław Windakiewicz. Windakiewicz svolse ricerche negli archivi di Padova, Bologna, Venezia e Siena, il cui frutto furono pubblicazioni in periodici e in volume di studi nonché di relative fonti storiche ${ }^{5}$. L'articolo Nacja polska w Padwie del 1887 costituisce un ampio studio concernente l'istruzione accademica della nobiltà polacca tra il ' 500 e il ' 600 , quando Padova si presentava come principale meta dei viaggi dei giovani polacchi. Lo studioso spiega le modalità di funzionamento dell'Università, descrive la vita studentesca, in particolar modo le forme dell'organizzazione delle comunità straniere nelle cosiddette nationes, con maggior attenzione rivolta alla natio polacca. Il tema viene ripreso nell'articolo del 1891, in seguito apparso anche in volume lo stesso anno. Il testo, che conta un centinaio di pagine, diviso in due parti, presenta una breve storia dell'Università padovana, della sua struttura e del funzionamento, focalizzando poi lo sguardo sulla presenza degli studenti polacchi. Windakiewicz, fornendo numerosi nomi di studenti e professori e descrivendo la loro attività, non si limita solo ai fatti, ma cerca anche di analizzare le motivazioni che spingono i polacchi ad intraprendere gli studi nella città veneta e gli influssi e le implicazioni sulla vita culturale, sociale e politica polacca. Infatti scorge nell'Ateneo padovano un'inestimabile scuola di formazione per gli statisti, i diplomatici, gli scienziati, i medici e i letterati polacchi, una porta che rese possibile l'accesso della Polonia alla comunità delle nazioni europee. Lo studioso riprende ancora il tema nel 1922, stavolta in forma di articolo di carattere divulgativo stampato sul mensile Przeglad Warszawski. Agli scritti di Windakiewicz si riferisce Henryk Barycz nel 1927 sul quadrimestrale Minerwa Polska. Il suo Zarys historiografii Uniwersytetu Padewskiego è uno studio bibliografico in cui l'autore, pur apprezzando il contributo di Windakiewicz, nota tuttavia l'incom-

skiego (contributo al convegno „Polonia - Veneto: viaggi, contatti, scambi”, Breslavia, 15-16.10.2020). Vedi l'articolo L'artista e i "luoghi della memoria". Padova nelle illustrazioni di Aleksander Gierymski pubblicate sulla stampa nel prossimo numero di Italica Wratislaviensia.

5 Windakiewicz pubblicò diversi documenti della natio polacca nella rivista Archiwum do Dziejów Literatury i Oświaty w Polsce del 1888. 
pletezza delle fonti da lui pubblicate, il che avrebbe influito in modo negativo su diversi studi successivi i cui autori non attingevano alle fonti primarie limitandosi solo a quelle stampate dallo studioso. Per quanto siano fondate queste obiezioni, si deve ammettere che gli studi di Windakiewicz costituirono il fondamento del sapere sulle vicende padovane dei polacchi e diedero l'avvio a diverse ricerche riguardanti le relazioni polacco-padovane con l'attenzione rivolta al secolo d'oro della nostra cultura e all'importanza dei soggiorni padovani per la maturazione artistica dei grandi poeti del Rinascimento e del primo Barocco. Lo stesso Barycz segue la strada tracciata dallo studioso che lo porta alla pubblicazione, negli anni 1971-1972, dell'intero archivio della natio polacca. Sembra che gli studi di Windakiewicz possano aver contribuito anche alle ricerche del padre Jan Warchał, francescano polacco residente a Padova, promotore dei lavori per la fondazione della "cappella polacca" nella Basilica del Santo. Nel 1913 Warchał pubblicò l'articolo Żydzi na Uniwersytecie Padewskim, in cui le alterne vicende degli Ebrei nella città veneta vengono presentate in relazione all'Università e alle condizioni della vita studentesca. Infatti, gli scolari, a cui la scarsità di denaro non di rado rendeva la vita difficile, non esitavano a ricorrere alle banche degli Ebrei. Ma i rappresentanti della comunità ebraica locale, come anche di quelle straniere, appaiono pure tra gli iscritti, per lo più alla facoltà di medicina. L'autore spiega le difficoltà sorte nelle procedure delle iscrizioni per le differenze confessionali, i conflitti che ne scaturivano e le possibili soluzioni del problema. L'articolo è documentato dalle matricole ritrovate di alcuni ebrei polacchi.

Gli scritti di Windakiewicz vengono richiamati anche in testi di carattere divulgativo pubblicati su giornali e riviste, come p.es. l'articolo di Aleksander Janowski, Pamiatki polskie w Padwie, sul settimanale Ziemia del 1911. Il tema ha particolarmente interessato Jan Pietrzycki, autore di 9 testi, stampati in maggioranza sui quotidiani (1917, 1920a, 1920b, 1922a, 1922b, 1926, 1928, 1929, 1937). Pietrzycki descrive le tracce polacche nella Basilica del Santo e racconta dell'usanza studentesca di iniziare i nuovi iscritti, chiamata in polacco "otrzęsiny".

Nell'ambito della tematica polacca riappare più volte la questione della cappella polacca nella Basilica del Santo. Il settimanale po- 
lacco Kraj pubblicato a Pietroburgo stampa nel 1899 tre testi di Adam Weryha-Darowski che la riguardano. Infatti, negli ultimi anni dell' 800 la basilica fu sottoposta a lavori di restauro. In quell'occasione il sopranominato padre Warchał ottenne il consenso di raggruppare lapidi commemorative e altre tracce della presenza polacca in una cappella del deambulatorio. Il francescano riuscì a raccogliere fondi necessari per il restauro della cappella. L'esecuzione degli affreschi fu affidata all'artista Tadeusz Popiel e in seguito valutata molto bene dalla parte italiana, rappresentata da Camillo Boito, architetto che preparò i progetti del nuovo altare e della ferrata di chiusura. Weryha-Darowski descrive la storia dell'antico altare polacco fondato nel 1607 e delle alterne sorti dei ricordi polacchi nel tempio, sottolineando l'importanza del luogo per l'identità culturale e nazionale dei polacchi. Oltre alle descrizioni l'autore fornisce due illustrazioni degli affreschi nel corso dei lavori e i ritratti di Boito e di Popiel.

Il tema viene ripreso da Darowski l'anno successivo nel settimanale varsaviano Tygodnik Ilustrowany (Weryha-Darowski, 1900). L'articolo, per il suo contenuto, non differisce dai testi di Kraj, ma viene completato da 6 illustrazioni eseguite da Gierymski che rappresentano la veduta generale del tempio, l'antico altare polacco e frammenti degli affreschi di Popiel. L'altare e la inferriata non sono ancora pronti, ma secondo l'autore i lavori si sarebbero dovuti concludere per la Pasqua del 1900. Probabilmente si tratta però dell'anno 1901, in quanto l'articolo esce il 9 giugno del $1900^{6}$. La cappella viene in seguito menzionata in tutti i testi su Padova pubblicati negli anni successivi, anche se non ha più quel sapore di novità; essa diventa una delle mete obbligatorie dei turisti e dei pellegrini polacchi che visitano la città, come testimonia anche nel 1903 l'articolo di Adam Dobrowolski, sempre su Tygodnik Ilustrowany, ricordo di un pellegrinaggio a Roma che fa parte del ciclo Szkice włoskie.

Padova ritorna anche nei testi dedicati al culto di sant'Antonio senza richiami specifici alla storia polacca. Lo si vede nella poesia di Jachimowski del 1878 che abbiamo citato prima, nell'articolo di Czesław

6 Per le informazioni riguardanti la fondazione della cappella polacca vedi Lenart (2020), per l'esecuzione delle decorazioni in particolare Wrana (2020). 
Jankowski del 1892 e, soprattutto, nei testi pubblicati nel 1931 in occasione del settimo centenario della morte del Santo. Il mensile Rodzina Polska stampa l'articolo di Józef Czarnecki, breve biografia del Santo di tono patetico, e il quotidiano Dziennik Poznański sul numero 135 pubblica due testi: una breve biografia del Santo di Dionizy Królikowski e una corrispondenza da Padova di Jan Kilarski. Quest'ultimo articolo si distingue da altri testi dedicati a Padova per il suo tono, in cui una vera ammirazione si mescola ad una leggera ironia ed esprime le impressioni del turista, stanco del calore, disorientato dall'impenetrabile groviglio delle vie e deluso per non aver visto tutto ciò che intendeva visitare.

Miasto monumentalnego piękna i brzydoty i niezrozumiałego nieładu w rozwoju i układzie [...] W Padwie ostatecznie wie się, gdzie się jest, ale trudno odgadnąć, którędy iść dalej. Uprzejme informacje przechodniów zwykle skierowują w stronę przeciwną od pożądanej, a przejrzystego planu miasta dotychczas nie ma. [...] Długa, niepiękna ulica zatłumiona już wieczorną publiką. Promenada, przegląd, wystawa siebie. [...] Ponad tem wszystkim na rozlepionym na murze afiszu zarys kwadratowej czaszki Il Duce. (Kilarski, 1931) ${ }^{7}$

L'anniversario del Santo diede impulso anche allo scritto di Wacław Husarski, che merita particolare attenzione. Husarski pubblica su Tygodnik Ilustrowany due testi nei numeri 26 e 27 (Husarski, 1931a, 1931b). Nel primo l'autore non si limita al tema del Santo e - come di solito avveniva - alla descrizione delle tracce polacche, ma propone al pubblico una descrizione esauriente - per quanto sia possibile farlo in un periodico - dei monumenti padovani e del carattere della città, passando in seguito alla vita di sant'Antonio e alle celebrazioni. Il secondo è invece dedicato a una mostra d'arte religiosa aperta a Padova. Husarski, non senza un certo orgoglio, informa che la Polonia si era trovata tra le

7 "Città di una bellezza e bruttezza monumentale, un incomprensibile caos nella sua struttura e nel suo sviluppo [...] A Padova in fin dei conti si sa dove ci si trova, ma non si sa come proseguire. I suggerimenti gentili dei passanti di solito ti indirizzano verso la parte opposta a quella desiderata e non si riesce a trovare una mappa chiara e leggibile. [...] La strada lunga e poco bella affollata di passanti serali, passeggiate, ostentazione di sé [...] E sopra a tutto ciò sul manifesto attaccato al muro, la sagoma del teschio quadrato, il Duce.” (trad. J. M.). 
poche nazioni (Germania, Austria e Olanda) a cui erano state assegnate nell'ambito dell'esposizione sale separate e che detiene il primato con i suoi 175 artefatti, presentati a turno per la limitatezza dello spazio espositivo. Le opere d'arte esposte nella sala polacca erano state accolte bene dalla critica italiana e alcune addirittura in modo entusiastico, come p.es. l'altare di Jan Szczepkowski (che secondo l'autore avrebbe suscitato l'ammirazione di Marinetti), le stazioni della Via crucis di Bogna Krasnodębska-Gardowska, o la lampada di Wiktor Gontarczyk. Husarski fornisce una lunga lista di artisti polacchi presenti alla mostra e l'articolo è completato dalle fotografie di alcune opere.

Tra gli articoli menzionati sopra, undici apparvero su quotidiani e dodici su settimanali di Cracovia, Varsavia, Poznań e Pietroburgo. Si tratta di riviste di tematiche generali di tipo culturale o letterario, tranne il settimanale Ziemia, di profilo più specialistico, organo della Società Polacca per il Turismo. Un articolo appare su un quindicinale di carattere popolare, cinque su mensili, tra cui Przeglad Warszawski, Przeglad Polski e Przegląd Współczesny sono riviste di cultura e letteratura con ambizioni scientifiche, due su quadrimestrali (Minerwa Polska, Kwartalnik Poświęcony Badaniu Przeszłości Żydów w Polsce) anch'essi di spessore piuttosto scientifico e infine uno su Rodzina Polska, periodico cattolico di cultura. Ovviamente la natura degli articoli cambia tra i quotidiani e le riviste in conformità alle competenze dei lettori. Mentre i giornali ospitano testi divulgativi nella rubrica feuilleton e a volte brevi note informative, i settimanali pubblicano articoli più esaurienti di dimensioni più ampie e non di rado fornite di illustrazioni. Nei mensili e nei quadrimestrali appaiono invece testi scientifici.

Gli autori degli articoli scientifici sono eminenti studiosi che non necessitano di ulteriori presentazioni: Stanisław Windakiewicz (1863-1943), polonista, professore dell'Università Jagellonica, membro dell'Accademia delle Scienze (Polska Akademia Umiejętności) e della Società Scientifica Varsaviana (Warszawskie Towarzystwo Naukowe); Henryk Barycz (1901-1994), storico, professore della Jagellonica, per diversi anni Direttore dell'Archivio dell'Università e membro di accademie scientifiche polacche (PAU, PAN). Sui quotidiani scrissero in generale autori interessati alle tematiche culturali italiane, anche se non 
necessariamente in senso professionale. Dionizy Królikowski (1862-1936), che pubblicò su Dziennik Poznański un testo in occasione delle celebrazioni di sant'Antonio, fu giornalista, inviato all'estero durante la guerra balcanica del 1912 e 1913, slavofilo, traduttore di letterature slave; Jan Kilarski (1882-1951), autore dell'articolo stampato sullo stesso quotidiano, fu di professione fisico e matematico, professore del Politecnico di Danzica, ma storico di questa città per passione e autore di numerose guide turistiche. Il più fecondo, Jan Pietrzycki (1880-1944), autore di 9 testi, di cui 6 stampati su quotidiani, fu giornalista, poeta e storico di letteratura legato a Leopoli, Danzica e Cracovia. Insegnante di ginnasio, molto attivo nel lavoro di carattere divulgativo e pedagogico, si interessava alle relazioni culturali italo-polacche svolgendo ricerche durante i suoi frequenti viaggi in Italia senza tuttavia produrre studi scientificamente validi. Collaborando con quotidiani per lo più cracoviani di diverso profilo ideologico (come Naprzód e Nowa Reforma di stampo socialista, Głos Narodu, periodico di destra e antisemita o il nazionalista Goniec Krakowski), aveva l'abitudine di riproporre più volte lo stesso scritto in forma quasi identica (prassi piuttosto comune nella stampa di allora), p. es. l'articolo sul rito dell'iniziazione studentesca del 1917 viene ristampato due volte nel 1920. Lo stesso succede con l'articolo Przybąźmy na czas, stampato due volte nel 1922 e poi, con poche modifiche e ampliamenti, riproposto nel 1926, 1928 e 1937.

Tra gli autori dei testi pubblicati sui settimanali troviamo Wacław Husarski, pittore, storico d'arte e critico artistico innamorato dell'Italia, traduttore di Dante; Adam Weryha-Darowski (1851-1911), pubblicista e storico a livello divulgativo, residente a Roma dal 1894 fino alla morte, dove svolgeva ricerche nell'Archivio Vaticano collaborando con numerose riviste polacche; Adam Dobrowolski (1860-1921), critico letterario e teatrale; Aleksander Janowski (1866-1944), geografo, pedagogo e viaggiatore in tutti i continenti, fondatore della Società Polacca per il Turismo (PTT) e suo presidente onorario a vita, nonché iniziatore della rivista Ziemia su cui pubblicò l'articolo menzionato prima. Come risulta dall'elenco presentato, Padova, soprattutto come luogo importante per la cultura polacca, fu oggetto d'interesse di autori la cui formazione 
professionale li indirizzava a questa tematica o di persone appassionate di viaggi e di turismo.

Soffermiamoci ora un attimo sugli articoli pubblicati nel 1922, anno in cui ricorreva il settecentesimo anniversario della fondazione dell'Università. Il testo di Jan Pietrzycki Przybądźmy na czas (Cerchiamo di arrivare in tempo), pubblicato il 3 e il 18 febbraio del 1922, ricorda le celebrazioni padovane progettate per i primi di maggio. Pietrzycki nota che la diplomazia polacca non aveva partecipato in modo soddisfacente alle celebrazioni del tricentenario della nascita di Molière svoltesi a Parigi nel gennaio dello stesso anno. Ora, scrive il giornalista, non dobbiamo lasciarci sfuggire l'occasione di segnare la nostra presenza in quell'Università con cui abbiamo sempre avuto tante relazioni.

Po tem, co stało się obecnie w Paryżu poważną troską napawa zbliżające się, a zapowiedziane na pierwsze dni maja siedemsetlecie uniwersytetu padewskiego, tego Nestora wszechnic europejskich. Będzie to uroczystość o ogromnem światowo-kulturalnem znaczeniu. Polska w obchodzie tym nie tylko, że winna wziąć udział, lecz zapewnić sobie należne jej, jedno z pierwszych stanowisk. (Pietrzycki, 1922a) ${ }^{8}$

Sfogliando diversi giornali di quell'anno non sono però riuscita a trovare notizie su come si presentava la partecipazione polacca, o in generale che forma avevano assunto le celebrazioni padovane. Considerando che l'Università padovana e la sua importanza per la cultura polacca costituiscono uno dei temi principali dei testi a stampa dedicati alla città veneta, può stupire il fatto che non sia stato possibile ritrovare nessuna relazione sulle celebrazioni dell'anniversario, tranne una breve informazione sul fatto che Stanisław Lencewicz, professore dell'Università di Varsavia, dopo la Riunione Internazionale Astronomico-geodetico-geofisica tenutasi a Roma il 2 maggio, si era recato a Padova per partecipare al Giubileo. Per quanto non si possa escludere che qual-

8 "Dopo quello che ora è successo a Parigi ci viene una grande ansia a pensare alle imminenti celebrazioni del settimo centenario dell'Università di Padova, tra i più antichi atenei europei, previste per i primi di maggio. Sarà un evento solenne di enorme significato culturale a livello mondiale. La Polonia non solo ci dovrebbe prendere parte, ma dovrebbe garantirsi una posizione di rilievo.” (trad. J. M.) 
che testo stampato su un quotidiano sia sfuggito nel corso della ricerca, è innegabile che le più importanti riviste di cultura sembrano non aver notato l'evento. L'anniversario dell'Università padovana venne solo menzionato in un breve testo di Windakiewicz, $W$ rocznice padewska 1222-1922, nel $1^{\circ}$ volume (maggio/giugno) di Przeglad Wspótczesny. L'autore non parla tuttavia dello svolgimento delle celebrazioni; dà solo basilari informazioni sull'A teneo come luogo di destinazione degli scolari polacchi. Il suo scritto precede il testo di Zdzisław Morawski, $U$ kolebki Uniwersytetu Padewskiego, che continua nel secondo volume della stessa annata. L'intervento di Morawski ha poco a che fare con l'Università e costituisce invece un ampio panorama della storia medievale del Nord Italia, con lo sguardo focalizzato su Padova, dei complessi conflitti tra i guelfi e i ghibellini, gli interventi degli imperatori svevi e infine le vicende della tirannia di Ezzelino da Romano. Nello stesso anno Windakiewicz prende la parola ancora una volta con il già menzionato articolo Polacy w Padwie pubblicato su Przeglad Warszawski. In quindici pagine lo studioso presenta un riassunto dei suoi studi ottocenteschi, dando ancora una volta un ampio quadro della presenza polacca all'Università e nella città veneta. Alla commemorazione polacca dell'anniversario padovano allude ancora Henryk Barycz nello scritto del 1927, affermando che esso aveva dato un nuovo impulso agli studi polacchi della tematica e menzionando il volume Omaggio dell'Accademia polacca di scienze e lettere all'Università di Padova nel settimo centenario della sua fondazione pubblicato a Cracovia nel 1922.

Come si vede da questa breve rassegna dei testi giornalistici dedicati a Padova, la loro tematica è legata in gran parte alle presenze polacche nella vita padovana. Confrontando questo approccio con le relazioni riguardanti altre città e regioni italiane, si può notare facilmente questo tratto specifico. Mentre, ad esempio, Roma appare soprattutto come centro del cattolicesimo e culla della civiltà europea con il suo tesoro di monumenti antichi, Milano viene presentata come capitale dell'opera mondiale, sede di famosi impresari e mercato degli artisti operistici oppure centro della moda ${ }^{9}$ e Assisi inevitabilmente viene ricondotta alla figura

9 A questo proposito si può citare p.es. Waleria Marrené $(1879,1882)$. 
di san Francesco, Padova, associata anche alla figura di sant'Antonio, diventa un mito e ritorna soprattutto come punto di riferimento culturale che nei secoli passati aveva contribuito alla formazione dell'élite intellettuale polacca. A partire dagli ultimi decenni del XIX sec. la stampa è uno strumento importante per plasmare le coscienze delle società e in Polonia negli anni della schiavitù e in quelli della nascita del nuovo stato indipendente si inscrive vistosamente nel lavoro volto a salvaguardare e ricostruire l'identità culturale della nazione. A questo scopo serve richiamare fonti e modelli "positivi e nobili" del secolo d'oro della cultura polacca e riproporre "luoghi di memoria" (Nora, 1984), carichi di sensi simbolici, la cui illustrazione visiva è costituita dai disegni di Aleksander Gierymski in precedenza menzionati. Non senza importanza sembra anche il richiamo all'identità religiosa, ritenuta all'epoca una componente significativa dell'identità nazionale.

\section{BIBLIOGRAFIA}

[-] (1895). Kurier Codzienny, 360, 1.

Barycz, H. (1927). Zarys historiografii Uniwersytetu Padewskiego. Minerwa Polska, 4, 311-323.

Czarnecki, J. (1931). 700-lecie św. Antoniego. Rodzina Polska, 6, 101-103.

Dobrowolski, A. (1903). Szkice włoskie - Padwa. Tygodnik Ilustrowany, 21, 407.

Husarski, W. (1931a). Città del Santo (Padwa w 700-lecie śmierci św. Antoniego Padewskiego). Tygodnik Ilustrowany, 26, 502-503.

Husarski, W. (1931b). Polska na wystawie sztuki religijnej w Padwie. Tygodnik Ilustrowany, 27, 522-523.

Jachimowski, W. (1878). Wspomnienia z pielgrzymki rzymskiej 1877. Pamiątka z Padwy. Chata, 12, 180-182.

Jankowski, C. (1892). San Antonio di Padova (z notatek turysty). Kurier Warszawski, 109, 1-2.

Janowski, A. (1911). Pamiątki polskie w Padwie. Ziemia, 39, 638-640; 40, 651-654.

Kilarski, J. (1931). W mieście św. Antoniego. Dziennik Poznański, 131, 2.

Królikowski, D. (1931). 700-lecie zgonu wielkiego świętego. Dziennik Poznański, 131, 3. 
Lenart, M. (Ed.). (2020). Polacy przy grobie św. Antoniego w Padwie. Czesść II - Kaplica polska (1896-2018). Opole: Uniwersytet Opolski.

Marrené, W. (1879). Cantopolis (Mediolan). Tygodnik Ilustrowany, 159, 2830.

Marrené, W. (1882). Listy z Włoch. Nowiny, 252, 1; 253, 1.

Miszalska, J. (2020). La stampa e la mediazione interculturale. Letteratura italiana sul settimanale Prawda (1881-1915). In N. Di Nunzio, \& A. Sciacovelli (Eds.), In viaggio, incontri, percezioni e riflessioni lungo il filo della poiesi (pp. 139-154). Warszawa: DiG.

Morawski, Z. (1922). U kolebki Uniwersytetu Padewskiego. Przeglad Współczesny, 1, 139-156; 2, 30-50.

Nora, P. (1984). Les Lieux de mémoire. Paris: Gallimard.

Pietrzak, P. (2017). Powiesść w świecie prasy. Bolesław Prus. Warszawa: PWN. Pietrzycki, J. (1917). Padewskie "otrzęsiny". O zwyczajach na uniwersytecie. Nowa Reforma, 277, 1.

Pietrzycki, J. (1920a). Jak Polacy “otrząsali się” w Padwie. Goniec Krakowski, 27, 3-4.

Pietrzycki, J. (1920b). Polonica padewskie. Naprzód, 248, 3-4.

Pietrzycki, J. (1922a). Przybądźmy na czas. Z powodu 700-letniego jubileuszu

Uniwersytetu w Padwie. Ilustrowany Kurier Codzienny, 34, 3.

Pietrzycki, J. (1922a). Przybądźmy na czas. Świat, 7, 8.

Pietrzycki, J. (1926). Pamiątki polskie w Padwie. Kurier Literacko-Naukowy, 30, III-IV.

Pietrzycki, J. (1928). Wspomnienia polskie w Padwie. Głos Narodu, 210, 4.

Pietrzycki, J. (1929). Wspomnienia polskie w Padwie. Tęcza, 18.

Pietrzycki, J. (1937). Co po Polakach zostało w Padwie? (z luźnych notatek). Polonia (Katowice), 4672, III.

Przeździecki A. (1853). O Polakach w Bononii i Padwie. Warszawa: Druk. Gazety Codziennej.

Pług, A. (1885) Polacy w Akademii Padewskiej. Kłosy, 26.02, 140-142.

Polewka C. (1887). Listy włoskie. Kłosy, 1136, 219-222.

Warchał, J. (1913). Żydzi polscy na uniwersytecie padewskim. Kwartalnik Poświęcony Badaniu Przeszłości Żydów w Polsce, 3, 37-72.

Weryha-Darowski, A. (1899a). Kaplica polska w Padwie w bazylice św. Antoniego. Kraj. Dziat Ilustrowany, 23, 30-31.

Weryha-Darowski, A. (1899b). Dwie polskie kaplice. Kraj. Dziat Ilustrowany, $50,347$. 
Weryha-Darowski, A. (1899c). Kaplica polska w Padwie. Kraj. Dziat Ilustrowany, 52, 369-373.

Weryha-Darowski, A. (1900). Kaplica polska w Padwie. Tygodnik Ilustrowany, 23, 450-452.

Windakiewicz, S. (1887). Nacja polska w Padwie. Przeglad Polski, 3, 460-492 .

Windakiewicz, S. (1891). Padwa. Studium z dziejów cywilizacji polskiej. Przegląd Polski, 99, 258-298; 548-597.

Windakiewicz, S. (1922a). Polacy w Padwie. Przeglad Warszawski, 10, 5-21.

Windakiewicz, S. (1922b). W rocznicę padewską 1222-1922. Przegląd Wspótczesny, t. 1, 133-138.

Wrana, M. (2020). Dekoracja malarska kaplicy polskiej pędzla Tadeusza Popiela - konkurs i realizacja (1898-1899). In M. Lenart (Ed.), Polacy przy grobie św. Antoniego w Padwie. Część II - Kaplica polska (1896-2018) (pp. 77-132). Opole: Uniwersytet Opolski.

Riassunto: L'Italia vista come culla della cultura europea e l'obiettivo delle peregrinazioni degli intellettuali e artisti polacchi appare spesso sulla stampa polacca degli ultimi decenni del XIX e dei primi del XX secolo. Tra le città descritte Padova non costituisce un tema così frequente come Venezia o Roma. Gli articoli dedicati a questa città hanno tuttavia un carattere particolare perché "Padova la dotta", oltre ad essere famosa per il culto di sant'Antonio, è presentata il più spesso nel contesto di multisecolari relazioni italo-polacche legate per lo più all'Università e quello dei polonica padovani. Due momenti inoltre trovano una risonanza particolare: il 1922 per il settimo centenario dell'Università e il 1931 per le celebrazioni del settimo centenario della morte del Santo. L'intervento propone la rassegna degli articoli apparsi su diversi periodici con una breve presentazione degli autori: studiosi, artisti o giornalisti attivi nella promozione della cultura italiana. Dai testi pubblicati sia nei quotidiani che sulle riviste di cultura o addirittura periodici di tipo scientifico emerge l'immagine della città fortemente segnata dalla presenza dei polacchi: studenti, professori dell'Università o pellegrini.

Parole chiave: Padova, università, periodici polacchi, relazioni italo-polacche, Basilica di Sant'Antonio 